For the excluded volume effect we have in the first approximation:

$$
\Delta m=\alpha m c
$$

where $\alpha$ determines the excluded volume (in the present units it equals the mass of solution in $\mathrm{kg} / \mathrm{g}$ polymer, from which the permeable electrolyte is excluded).

Comparing equation (2) with the experimental results, we see that with POE the agreement is excellent. From both diagrams we obtain $\alpha=0.0027 \mathrm{~kg} / \mathrm{g}$. As the polymer mass itself is included in $\alpha$ this means that for each gram of the polymer there are $1.7 \mathrm{~g}$ of (solvating) water from which sodium hydroxide is excluded. With PMA the situation is more complicated. A comparison of the experimental results in Fig. 2 with the predictions of the Donnan theory and the excluded volume effect reveals that the experimental curve may be obtained by a suporposition of these two effects. It is interesting to note that the excluded volume effect for PMA happens to be of the same magnitude as for POE.

I may conclude by stating that the experiments recorded here indicate that in linear polyelectrolyte solu tions two effects are present, namely, the classical Donnan effect and the excluded volume effect. The latter occurs even with non-electrolyte polymers. These matters are treated further in a paper to be published in Acta Chemica Scandinavica.

Institute of Physical Chemistry,

H. VINK

University of Uppsala.

${ }^{1}$ Donnan. F. G., Z. Electrochem., 17, 572 (1911).

${ }^{2}$ Harris, F. E., and Rice, S. A., J. Phys. Chem., 58, 725 (1954)

${ }^{3}$ Hill, T. L., Farad. Soc. Disc., 21, 31 (1956).

4 Stigter, D., and Hill, T. I., J. Phys. Chem., 63, 551 (1959).

${ }^{5}$ Orofino, T. A., and Flory, P. J., J. Phys. Chem., 63, 283 (1959).

' Stigter, D., J. Phys. Chem., 64, 838 (1960).

"Strauss, U. P., and Ander, P., J. Amer. Chem. Soc., 80, 6494 (1958)

\section{Partial Differentiation, with Special Reference to Thermodynamics}

From time immemorial savants and scholars have taken liberties with well-known words and phrases and by 'new definitions' have imparted highly specialized meanings to familiar expressions. While in some cases such actions can be justified on the grounds of greater clarity of meaning and greater precision of definition, as a general practice the imparting of specialized meanings to wellknown words and phrases tends to obscure any language, even the language of mathematics, particularly when the specialized meanings involve largely meaningless 'new definitions'.

In a recent communication, Elliott ${ }^{1}$ has suggested a new definition for the partial derivatives occurring in thermodynamics. The approach is based on the form:

$$
\mathrm{d} z=a \mathrm{~d} x_{1}+b \mathrm{~d} x_{2}+c \mathrm{~d} x_{3}
$$

and it is stated that $a, b$, and $c$ in the limit of small displacement $\mathrm{d} x_{1}, \mathrm{~d} x_{2}, \mathrm{~d} x_{3}$ are to be identified with the new partial derivatives and indicated as $\frac{\partial z}{\partial x_{1}}$, etc.

No restrictions are imposed on the manner in which the limit is to be taken and, in our opinion, the failure to do this renders the definition meaningless.

As an illustration of our criticism let us introduce the symbols $Z_{1}, Z_{2}, Z_{3}$ to represent the 'old' derivatives of $z$, that is, $Z_{1}$ is the rate of change of $z$ with respect to $x_{1}$ keeping $x_{2}$ and $x_{3}$ constant. Then to a first order of small quantities:

$$
\mathrm{d} z=Z_{1} \mathrm{~d} x_{1}+Z_{2} \mathrm{~d} x_{2}+Z_{3} \mathrm{~d} x_{3}
$$

Evidently by keeping $x_{2}$ and $x_{3}$ constant we can deduce from (1) and (2) that:

$$
a=\frac{\partial z}{\partial x_{1}}=Z_{1}
$$

On the other hand, for $\mathrm{d} x_{1}=\mathrm{d} x_{2}$ and $x_{3}$ kept constant

$$
\frac{\partial z}{\partial x_{1}}=Z_{1}+Z_{2}
$$

and so on. The symbol $\frac{\partial z}{\partial x_{1}}$ can have an infinity of values depending on how we approach the limit.

Dr. Elliott implies that the freedom from restrictions in taking the limit is one of the merits of his definition. We believe it can only lead to confusion in a field where precise definitions are essential.

\section{G. C. Benson}

E. A. FLOOD

Division of Pure Chemistry,

National Research Council of Canada,

$$
\text { Ottawa. }
$$

${ }^{1}$ Elliott, G. A., Nature, 198, 1191 (1963).

THE preceding communication shows that if the partial differential indicated by $\partial z / \partial x_{1}$ is defined as the limiting value of the rate of change of $z$ with respect to $x_{1}$, and if we include changes due to the variation of $x_{2}$ (which is dependent on $x_{1}$ ) then the result obtained depends on the relation between $x_{1}$ and $x_{2}$ and in particular if $\mathrm{d} x_{1}=\mathrm{d} x_{2}$ the expression is:

$$
\partial z / \partial x_{1}=Z_{1}+Z_{2}
$$

This 'definition' is therefore quite useless, and on this point there is no dispute.

On the other hand, if we define the partial differential in the way proposed in my previous communication we always obtain the result $\partial z / \partial x_{1}=a$ and no other answer is possible. The symbol $\partial z / \partial x_{1}$ is to be regarded simply as a rather clumsy but time-honoured method of indicating the coefficient $a$ or rather its limiting value when the neglect of differentials of higher order than the first in forming equation (1) above is strictly justified. The new definition is strictly independent of any relations between the variables.

\section{G. A. Elliotot}

Department of Chemistry,

University of Western Australia, Perth.

\section{BIOCHEMISTRY}

\section{Enzymatic Evidence for the Configuration of d-Isocitric Acid}

THe configuration of the $\alpha$-carbon atom of the $d$-isocitric acid of the Krebs tricarboxylic acid cycle was designated $\mathrm{L}_{\mathrm{S}}$ on the basis of an application of the Lovene displacement rule ${ }^{1}$, but subsequent chemical ${ }^{2-4}$ and X-ray ${ }^{5}$ studies indicated the $\mathrm{D}_{\mathrm{s}}$ configuration. Other investigations have shown that the $\alpha$ - and $\beta$-asymmetric centres of $d$-isocitric acid possess opposite configurations ${ }^{6,7}$. Although $d$-isocitric acid may be distinguished by the action of isocitric dehydrogenase from the three isomeric isocitric acids, none of the isomers of $\alpha$-aminotricarballylic acid, the $\alpha$-amino analogue of isocitric acid, has thus far been found to be attacked by enzymes. Groenstein and Winitz ${ }^{1}$, who prepared both DL- $\alpha$-aminotricarballylic and DL-allo- $\alpha$ aminotricarballylic acids, were unable to find an enzyme capable of resolving these and therefore resorted to the use of brucine. They thus obtained tho corresponding four optical isomers, which they converted to the respective isocitric acids by treatment with $\mathrm{HNO}_{2}$.

Application of the Clough-Lutz-Jirgensons rule permitted assignment of configuration to the $\mathrm{D}$ - and $\mathrm{L}$-isomers of allo- $\alpha$-aminotricarballylic acid, but assignment of the configurations of the isomers of $\alpha$-aminotricarballylic 